United Nations Educational Scientific and Cultural Organization and International Atomic Energy Agency

\title{
CODIMENSION TWO KÄHLER SUBMANIFOLDS OF SPACE FORMS
}

\author{
M.J. Ferreira ${ }^{1}$ \\ Departamento de Matemática, Faculdade de Ciências, Universidade de Lisboa, \\ R. Ernesto de Vasconcelos, C1, 30, 1749-016, Lisboa, Portugal \\ and \\ R. Tribuzy ${ }^{2}$ \\ Universidade do Amazonas, Instituto de Matemática, ICE, \\ 6900, AM, Manaus, Brasil \\ and \\ The Abdus Salam International Centre for Theoretical Physics, Trieste, Italy.
}

\begin{abstract}
In this article we study isometric immersions from Kähler manifolds whose $(1,1)$ part of the second fundamental form is parallel, the ppmc isometric immersions. When the domain is a Riemann surface these immersions are precisely those with parallel mean curvature. P. J. Ryan has classified the Kähler manifolds that admit isometric immersions, as real hypersurfaces, in space forms. We classify the codimension two ppmc isometric immersions into space forms.
\end{abstract}

MIRAMARE - TRIESTE

March 2001

\footnotetext{
${ }^{1}$ E-mail: mjferr@ptmat.lmc.fc.ul.pt

${ }^{2}$ E-mail: tribuzy@buriti.com.br
} 


\section{Introduction and Statement of Results}

Surfaces with parallel mean curvature play an interesting part in the study of the geometry of submanifolds. The complex structure of such a surface is a crucial ingredient in the building of this theory. One may ask: which Kähler candidates generalize the parallel mean curvature surfaces?

To study the extrinsic geometry of an isometric immersion $\varphi: M \rightarrow N$ between two Riemannian manifolds, one of the main tools is its second fundamental form $\alpha=\nabla d \varphi$. In such study, when $M$ is Kähler, its complex structure $J$ fulfil an important role.

The complexified $\alpha$ splits in a natural way according to types giving rise to

$$
\alpha=\alpha^{(1,1)}+\alpha^{(2,0)}+\alpha^{(0,2)}
$$

When $\alpha^{(1,1)} \equiv 0, \varphi$ is called pluriminimal or $(1,1)$ - geodesic. Equivalently $\varphi$ is pluriminimal if and only if $\varphi_{\mid C}$ is minimal for any holomorphic curve $C \subset M$. Pluriminimal isometric immersions have been studied by several authors (see for instance [3], [4], [5]).

When $N$ has non positive curvature operator, every minimal isometric immersion is pluriminimal [4]. Clearly, if $\operatorname{dim} M=2$, the concepts of minimality and pluriminimality coincide.

Surfaces with parallel mean curvature generalize in a natural way the minimal ones. They can be characterized by the parallelism of the $(1,1)$ - part of their second fundamental form. So, in a higher dimensional setting the isometric immersions with parallel $\alpha^{(1,1)}$ generalize the parallel mean curvature surfaces. One of the fundamental questions is to know which Kähler manifolds admit isometric immersions with $\alpha^{(1,1)}$ parallel.

We will say that an isometric immersion from a Kähler manifold is a parallel pluri-mean curvature immersion (ppmc) if $\alpha^{(1,1)}$ is parallel. It is easily seen that $\varphi$ is ppmc if and only if $\varphi_{\mid C}$ has parallel mean curvature for any totally geodesic holomorphic curve $C \subset M$.

$P P M C$ isometric immersions into space-forms display, indeed, some special features of the parallel mean curvature surfaces, namely the existence of a 1-parameter deformation through a smooth family of ppmc isometric immersions which, up to a parallel isomorphism, have the same normal bundle [7].

The aim of the present work is to classify the, non minimal, codimension two ppmc isometric immersions into space-forms. It turns out that if these immersions do not factorize, they are either surfaces with parallel mean curvature or minimal submanifolds of horospheres in an hyperbolic space. This is not true for codimension three as it shows the emdedding of $C P^{2}$ into the unit sphere $S^{7}$ in the Lie algebra of $S U(3)$.

Kähler hypersurfaces into space-forms have been studied by Ryan, P. J. [12], who presented the following results:

Theorem 1 [12] Let $M$ be a Kähler hypersurface of a space-form with sectional curvature $c \neq 0$ and $\operatorname{dim} 2 m+1$. Then, when $m>1, M$ is an open set of one of the following manifolds: 
- $(c>0) S^{2}\left(c_{1}\right) \times S^{2}\left(c_{2}\right) \subset S^{5}(c)$, where $S^{k}(r)$ denotes the $k$-dimensional Euclidean sphere with constant sectional curvature $r$ and $\frac{1}{c_{1}}+\frac{1}{c_{2}}=\frac{1}{c}$.

- $(c<0)$ a horoshere in $H^{2 m+1}(c)$ or $H^{2}\left(c_{1}\right) \times S^{2}\left(c_{2}\right) \subset H^{5}(c)$, where $H^{k}(r)$ denotes the $k$-dimensional hyperbolic space with constant sectional curvature $r$ and $\frac{1}{c_{1}}+\frac{1}{c_{2}}=\frac{1}{c}$.

We remark that all the submanifolds given in the previous theorem provide examples of ppmc isometric immersions.

Theorem 2 [12] Let $M$ be a Kähler hypersurface of $R^{2 m+1}$. Then $M$ is either an open subset of a Riemann surface $S$ or an open set of the Riemannian product $S \times R^{2 m-1}$.

Corollary 3 Let $M$ be a Kähler manifold with complex dimension $m$ and $\varphi: M \rightarrow R^{2 m+1} a$ ppmc isometric immersion. Then, locally, one of the following conditions occur:

- $m=1$ and $\varphi: M \rightarrow R^{3}$ has constant mean curvature.

- $M=M_{1} \times M_{2}$ and $\varphi=\varphi_{1} \times \varphi_{2}: M_{1} \times M_{2} \rightarrow R^{3} \times R^{2 m-2}$, where $M_{1}$ is a Riemann surface, $\varphi_{1}$ has parallel mean curvature and $\varphi_{2}$ is totally geodesic.

Regarding codimension two isometric immersions from a Kähler manifold with complex dimension $m$, we have the following results:

Theorem 4 Assume that $\varphi: M \rightarrow S^{2 m+2}(c)$ is a, non minimal, ppmc isometric immersion. Therefore one of the following conditions hold:

- $m=1$ and $\varphi: M \rightarrow S^{4}(c)$ has parallel mean curvature.

- $m=2, M$ is an open subset of $S^{2}\left(c_{1}\right) \times M_{2}$ and $\varphi=\varphi_{1} \times \varphi_{2}: M \rightarrow S^{2}\left(c_{1}\right) \times S^{3}\left(c_{2}\right)$, where $\varphi_{1}: S^{2}\left(c_{1}\right) \rightarrow R^{3}$ is the standard embedding, $\varphi_{2}: M_{2} \rightarrow S^{3}\left(c_{2}\right)$ is minimal and $\frac{1}{c_{1}}+\frac{1}{c_{2}}=\frac{1}{c}$.

- $m=3, M$ is an open subset of $S^{2}\left(c_{1}\right) \times S^{2}\left(c_{2}\right) \times S^{2}\left(c_{3}\right), \frac{1}{c_{1}}+\frac{1}{c_{2}}+\frac{1}{c_{3}}=\frac{1}{c}$ and $\varphi=h_{\mid M}$, where $h: S^{2}\left(c_{1}\right) \times S^{2}\left(c_{2}\right) \times S^{2}\left(c_{3}\right) \rightarrow S^{8}(c)$ is the standard emdedding.

Theorem 5 Assume that $\varphi: M \rightarrow H^{2 m+2}(c)$ is a, non minimal, ppmc isometric immersion. Then one of the following conditions hold:

- $m=1$ and $\varphi: M \rightarrow H^{4}(c)$ has parallel mean curvature.

- $m>1, \varphi(M) \subset E^{2 m+1} \subset H^{2 m+2}(c)$ and $\varphi: M \rightarrow E^{2 m+1}(c)$ is minimal.

- $M$ is locally a Riemannian product, $M_{1} \times M_{2}, \varphi(M) \subset E^{2 m+1}$ and $\varphi=\varphi_{1} \times \varphi_{2}: M \rightarrow$ $E^{2 m+1}$ is a product immersion, where $M_{1}$ is a Riemann surface and $\varphi_{2}$ is totally geodesic. 
- $M$ is locally a Riemannian product $M_{1} \times M_{2}$, where $M_{2}$ is an open subset of $H^{2}\left(c_{2}\right)$; moreover $\varphi=\varphi_{1} \times \varphi_{2}: M_{1} \times M_{2} \rightarrow S^{3}\left(c_{1}\right) \times H^{2}\left(c_{2}\right) \subset H^{6}(c)$, where $\varphi_{1}: M_{1} \rightarrow S^{3}\left(c_{1}\right)$ has constant mean curvature and $\frac{1}{c_{1}}+\frac{1}{c_{2}}=\frac{1}{c}$.

- $M$ is locally a Riemannian product $M_{1} \times M_{2}$, where $M_{2}$ is an open subset of $S^{2}\left(c_{2}\right)$; moreover $\varphi=\varphi_{1} \times \varphi_{2}: M_{1} \times M_{2} \rightarrow H^{3}\left(c_{1}\right) \times S^{2}\left(c_{2}\right) \subset H^{6}(c)$ where $\varphi_{1}: M_{1} \rightarrow H^{3}\left(c_{1}\right)$ has constant mean curvature and $\frac{1}{c_{1}}+\frac{1}{c_{2}}=\frac{1}{c}$.

- $M$ is an open subset of $H^{2}\left(c_{1}\right) \times S^{2}\left(c_{2}\right) \times S^{2}\left(c_{3}\right)$ and $\varphi=h_{\mid M}$ where $h: H^{2}\left(c_{1}\right) \times S^{2}\left(c_{2}\right) \times$ $S^{2}\left(c_{3}\right) \rightarrow H^{8}(c)$ is the standard embedding and $\frac{1}{c_{1}}+\frac{1}{c_{2}}+\frac{1}{c_{3}}=\frac{1}{c}$.

- $M$ is an open subset of $E^{2 r} \times S^{2}\left(c_{2}\right)$ and $\varphi=h_{\mid M}$, where $r=m-1, \frac{1}{c_{1}}+\frac{1}{c_{2}}=\frac{1}{c}, E^{2 r}$ is a horosphere in $H^{2 r+1}\left(c_{1}\right)$ and $h: E^{2 r} \times S^{2}\left(c_{2}\right) \rightarrow H^{2 m+2}(c)$ is the standard embedding.

Theorem 6 Let $\varphi: M \rightarrow R^{2 m+2}$ be a, non minimal, ppmc isometric immersion. Then one of the following conditions hold:

- $m=1$ and $\varphi: M \rightarrow R^{4}$ has parallel mean curvature.

- $m=2, M=M_{1} \times M_{2}$ and $\varphi=\varphi_{1} \times \varphi_{2}: M_{1} \times M_{2} \rightarrow R^{6}$, where $\operatorname{dim} M_{1}=\operatorname{dim} M_{2}=2$ and, for each $1 \leq i \leq 2, \varphi_{i}: M_{i} \rightarrow R^{3}$ has constant mean curvature.

- $M=M_{1} \times M_{2}$ and $\varphi=\varphi_{1} \times \varphi_{2}: M_{1} \times M_{2} \rightarrow R^{2 m+2}$ where $\varphi_{1}$ satisfies one of the two previous conditions and $\varphi_{2}$ is totally geodesic.

Isometric immersions with parallel second fundamental form are trivially ppmc. We shall remark that minimal isometric immersions from a Kähler manifold into a horosphere $E^{2 m+1} \subset$ $H^{2 m+2}$ provide examples of $p p m c$ isometric immersions which do not have parallel second fundamental form.

\section{Preliminaries}

Throughout this work all geometric objects are assumed to be smooth and $M$ will denote a connected Kähler manifold with comlex dimension $m$ and complex structure $J$.

Let $\varphi: M \rightarrow N$ be an isometric immersion into a Riemannian manifold. $\stackrel{\perp}{T} M$ will denote the normal bundle of $\varphi$. We will use the same symbol $\nabla$ to represent the Levi-Civita connection of $T M$ or the induced connection on $\varphi^{-1} T N$ and $T^{*} M \otimes \varphi^{-1} T N$. The symbol $\stackrel{\perp}{\nabla}$ will be used to represent either the induced connection on $\stackrel{\perp}{T} M$ or on $T^{*} M \otimes \stackrel{\perp}{T} M$.

The complex structure $J$ gives rise to the usual splitting

$$
T^{C} M=T^{\prime} M \oplus T^{\prime \prime} M
$$


of the complexified tangent bundle $T^{C} M$ into its holomorphic and anti-holomorphic parts. We will denote respectively by $\pi^{\prime}: T^{C} M \rightarrow T^{\prime} M$ and $\pi^{\prime \prime}: T^{C} M \rightarrow T^{\prime \prime} M$ the orthogonal projections with respect to the hermitian structure of $M$.

We have therefore

$$
\alpha^{(1,1)}(X, Y)=\alpha\left(X^{\prime}, Y^{\prime \prime}\right)+\alpha\left(X^{\prime \prime}, Y^{\prime}\right)
$$

where $Z^{\prime}=\pi^{\prime}(Z)$ and $Z^{\prime \prime}=\pi^{\prime \prime}(Z)$ for every $Z \in C\left(T^{C} M\right)$.

Similarly

$$
\alpha^{(2,0)}(X, Y)=\alpha\left(X^{\prime}, Y^{\prime}\right)
$$

we can also write

$$
\alpha^{(1,1)}(X, Y)=\frac{1}{2} C(X, Y)+\frac{i}{2} C(X, J Y)
$$

where

$$
C(X, Y)=\frac{1}{2}\{\alpha(X, Y)+\alpha(J X, J Y)\}
$$

and

$$
\alpha^{(2,0)}(X, Y)=\frac{1}{2} D(X, Y)-\frac{i}{2} D(X, J Y)
$$

where

$$
D(X, Y)=\frac{1}{2}\{\alpha(X, Y)-\alpha(J X, J Y)\}
$$

The operators $C$ and $D$ characterize the second fundamental form and play an interesting role in the study of immersions from Kähler manifolds.

As we have seen before, the vanishing of $C$ tells the minimality of the restriction of $\varphi$ to any holomorphic curve of $M$. In a similar way, the vanishing of $D$ means that the restriction of $\varphi$ to any holomorphic curve of $M$ is totally umbilic. These properties are rather special when $N$ is a space form. Under this assumption we have:

Proposition 7 [4] Let $\varphi: M \rightarrow Q(c)$ be an isometric immersion. Then

- if $c=0, \varphi$ is minimal if, and only if, $\varphi$ is pluriminimal

- if $c \neq 0$ and $\varphi$ is pluriminimal, $m=1$.

We say that $C$ is totally umbilic if $C=\langle\rangle$,$H , where H$ denotes the mean curvature of $\varphi$ and $\langle$,$\rangle is the Riemannian structure of M$.

Proposition 8 [9] Let $\varphi: M \rightarrow Q(c)$ be an isometric immersion with $C$ totally umbilic. Then

- if $c=0$, either $\varphi$ is minimal or $m=1$.

- if $c>0, m=1$.

- if $c<0$, either $m=1$ or $\varphi$ has constant mean curvature $\|H\|=\sqrt{-c}$.

Proposition 9 [10] Let $\varphi: M \rightarrow Q(c)$ be an isometric immersion. Then $D=0$ if and only if $\varphi$ has parallel second fundamental form. 


\section{3 ppmc Isometric Immersions}

ppmc isometric immersions were introduced in [7] and [8].

When $m=1$, ppmc isometric immersions are precisely those immersions with parallel mean curvature. It is well known that a parallel mean curvature surface in a three dimensional spaceform allows a one parameter family of isometric deformations - the associate family - with the same mean curvature. ppmc isometric immersions into a space-form $Q(C)$ also enjoy this property. Indeed, associated to $J$ there is a one parameter family

$$
J_{\theta}=\cos \theta I+\sin \theta J, \theta \in[0, \pi]
$$

of tensors, where $I$ denotes the identity map of $T M$.

For each $\theta$ we define $\alpha_{\theta} \in C\left(\stackrel{2}{\odot} T^{*} M \otimes \stackrel{\perp}{T} M\right)$ by

$$
\alpha_{\theta}(X, Y)=\alpha\left(J_{\frac{\theta}{2}}(X), J_{\frac{\theta}{2}}(Y)\right)
$$

$\alpha_{\theta}$ is a symmetric tensor field which satisfy Gauss, Codazzi and Ricci equations. Hence, from the fundamental theorem for submanifolds, we get an isometric immersion $\varphi_{\theta}: M \rightarrow Q(c)$ whose second fundamental form is $\alpha_{\theta}$.

ppmc isometric immersions are exactly characterized by the existence of an associated family.

Proposition 10 [8] Let $\varphi: M \rightarrow Q(c)$ be an isometric immersion. Then $\varphi$ is ppmc if, and only if, $\varphi$ has an associated family.

Remark 11 The hypothesis $(\stackrel{\perp}{R})^{(2,0)}$ considered in [8] is not necessary, as pointed out in [2]. It follows from the parallelism of $\alpha^{(1,1)}$.

Assume now that $M=M_{1} \times M_{2}$, where $M_{1}$ and $M_{2}$ are Kähler manifolds and $\varphi: M_{1} \times$ $M_{2} \rightarrow Q(c)$ is a codimension two ppmc isometric immersion. As a consequence of the previous proposition we can state:

Proposition 12 Under the above conditions, for each $a \in M_{1}$ (resp. $b \in M_{2}$ ), the immersion $\varphi^{a}: M_{2} \rightarrow Q(c)$ (resp. $\varphi^{b}: M_{1} \rightarrow Q(c)$ ) defined by $\varphi^{a}(y)=\varphi(a, y)\left(\operatorname{resp} . \varphi^{b}(x)=\varphi(x, b)\right)$ is ppmc and has flat normal bundle.

Proof. If $\varphi_{\theta}$ is an associated family of $\varphi, \varphi_{\theta}^{a}(y)=\varphi_{\theta}(a, y)$ is a smooth deformation of $\varphi^{a}$. Since the second fundamental form $\beta$ of $\varphi^{a}$ is obtained by the restriction of $\alpha$ to $T M_{2}$, it is easily seen that $\beta_{\theta}(X, Y)=\beta\left(J_{\frac{\theta}{2}}^{2}(X), J_{\frac{\theta}{2}}^{2}(Y)\right)$ is the second fundamental form of $\varphi_{\theta}^{a}$, where $J^{2}$ represents the complex structure of $M_{2}$. Thus $\varphi$ is ppmc.

For $\xi \in C\left(\stackrel{\perp}{T} M_{2}\right)$ we let $A_{\xi}$ denote the Weigarten operator for $\varphi_{a}$, corresponding to $\xi$. Clearly, if $v \in T_{a} M_{1} \subset \stackrel{\perp}{T} M_{2}, A_{v}=0$. Therefore, using Ricci equation for $\varphi_{a}$, we easily conclude that $\stackrel{\perp}{R}(.,) v=$.0 , where $\stackrel{\perp}{R}$ represents the normal curvature of $\varphi_{a}$. 
On the other hand, the fact that the mean curvature $H$ of $\varphi_{a}$ is a parallel section of $\stackrel{\perp}{T} M_{2}$ which sits on $\stackrel{\perp}{T} M$ allows us to conclude that, for every $\xi \in C(\stackrel{\perp}{T} M), \stackrel{\perp}{R}(.,.) \xi=0$, so that $\stackrel{\perp}{T} M_{2}$ is flat.

\section{Proof of the Main Results}

Assume that $\varphi: M \rightarrow Q(c)$ is a ppmc isometric immersion.

For each $\xi \in C(\stackrel{\perp}{T} M)$ we let $C_{\xi} \in C\left(T^{*} M \otimes T M\right)$ be the operator defined by

$$
C_{\xi}(X, Y)=\langle C(X, Y), \xi\rangle
$$

where $C$ was defined in section two. Equivalently

$$
2 C_{\xi}=A_{\xi}-J A_{\xi} J
$$

$C_{\xi}$ is a self-adjoint operator which commutes with $J$. It is clearly parallel whenever $\xi$ is a parallel normal section.

To prove theorems 4,5 and 6 we use the techniques for decomposing manifolds into products of integral manifolds corresponding to eigenspaces of convenient $C_{\xi}$.

For each $x \in M$, let $\lambda_{1}(x), \cdots, \lambda_{k}(x)$ be the eigenvalues of $C_{H}(x)$ and let $T_{x}^{1}, \cdots, T_{x}^{k}$ be the corresponding eigenspaces.

$$
T_{x} M=T_{x}^{1} \stackrel{\perp}{\oplus} \cdots \stackrel{\perp}{\oplus} T_{x}^{k}
$$

Since $C$ is parallel for $p p m c$ immersions, we obtain in this way parallel distributions $T^{1}, \cdots, T^{k}$ which are invariant under the action of the holonomy group $\operatorname{Hol}(g)$, where $g$ denotes the Riemannian structure of $M$. Moreover, each $T^{i}, 1 \leq i \leq k$, is $J$-invariant. From the de Rham theorem we know that $M$ is, locally, a product $\left(M_{1}, g_{1}\right) \times \cdots \times\left(M_{k}, g_{k}\right)$ and $\operatorname{Hol}(g)=\operatorname{Hol}\left(g_{1}\right) \times \cdots \operatorname{Hol}\left(g_{k}\right)$ where, for each $1 \leq i \leq k, \operatorname{Hol}\left(g_{i}\right)$ is a subgroup of $O(2 m)$ acting trivially on $T^{j}$ if $j \neq i$. We get then a foliation whose leaves are Kähler and totally geodesic manifolds.

Using $N$ to represent a unit normal vector field orthogonal to $H$, we consider now, for each $1 \leq i \leq k$, the operator $\widetilde{C}_{N}=p_{T_{i}} \circ C_{N}: T^{i} \rightarrow T^{i}$ which commutes also with $J$, is parallel and self-adjoint. Each $M_{i}$ decomposes again, locally, as a product $M_{i}^{1} \times \cdots \times M_{i}^{r_{i}}$ of Kähler manifolds, so that, after these two factorization steps, we end up with

$$
M=M_{1} \times \cdots M_{r},
$$

where each $M_{i}$ is Kähler. However, at this stage is not clear that the $T^{i}$ may be eigenspaces for $C_{N}$. This turns out to be true.

Since $\varphi$, by assumption, is not minimal we can select $i \in\{1, \cdots, r\}$ such that $\operatorname{trace} \alpha_{\mid T^{i} \times T^{i}} \neq$ 0 . To simplify we assume $i=1$ and consider $M=M_{1} \times \widetilde{M}$, where $\widetilde{M}=M_{2} \times \cdots M_{r}$.

We consider $a=\left(a_{1}, a_{2}\right) \in M_{1} \times \widetilde{M}$ and claim that $\alpha(v, w)=0$ whenever $v \in T_{a_{1}} M_{1}$ and $w \in T_{a_{2}} \widetilde{M}$. 
To prove the claim we fix $a_{2} \in \widetilde{M}$ and consider $\varphi_{a_{2}}: M_{1} \rightarrow Q(c)$. According to proposition $12 \varphi_{a_{2}}$ is ppmc and has flat normal bundle. It follows that its first normal bundle is parallel and we can reduce the codimension (cf.[6]) . Therefrom $\varphi_{a_{2}}\left(M_{1}\right)$ is contained in a totally geodesic submanifold $\widetilde{Q}(c)$ of $Q(c)$, where $\operatorname{dim} \widetilde{Q}(c) \leq \operatorname{dim} M_{1}+2$. The claim is now a consequence of the fact that the first normal bundle of $\varphi_{a_{2}}$ at $x$ coincides with the first normal bundle of $\varphi$ at $\left(x, a_{2}\right)$.

We proceed now analysing separately each of the three situations.

$\mathbf{c}=\mathbf{0} \quad$ We know from proposition 8 that $M_{1}$ must be a Riemann surface. It follows from Lemma $1[11]$ that $\varphi=\varphi_{1} \times \varphi_{2}$ is a product of immersions and two alternative possibilities come out:

1. $\varphi_{1}: M_{1} \rightarrow R^{4}$ has parallel mean curvature and $\varphi_{2}$ is totally geodesic.

2. $\varphi_{1}: M_{1} \rightarrow R^{3}$ and $\varphi_{2}: M_{2} \rightarrow R^{2 m-1}$ are two isometric immersions with constant mean curvature.; from corollary 3 we infer that either $\operatorname{dim} M_{2}=2$ or $M_{2}=\widetilde{M}_{2} \times \widetilde{M}$ and $\varphi=\rho \times \sigma$, where $\widetilde{M}_{2}$ is a Riemann surface, $\rho$ has parallel mean curvature and $\sigma$ is totally geodesic.

$\mathbf{c}>\mathbf{0}$ As above, we know from proposition 8 that $M_{1}$ is a surface. Applying Lemma 1 [11] to $\varphi: M_{1} \times \widetilde{M} \rightarrow R^{2 m+3}$ we conclude that $\varphi=\varphi_{1} \times \varphi_{2}$ is a product of ppmc immersions and three distinct situations may be considered:

1. $m=1$ and $\varphi: M \rightarrow S^{4}$ has parallel mean curvature.

2. $\varphi_{1}\left(M_{1}\right) \subset R^{4}$ and $\varphi_{2}$ has codimension 1. Then $\varphi_{1}\left(M_{1}\right) \subset S^{3}\left(c_{1}\right)$, and $\varphi_{1}: M_{1} \rightarrow S^{3}\left(c_{1}\right)$ has constant mean curvature. On the other hand, since $\varphi(M) \subset S^{2 m+2}$, it follows from propositon 8 that $\operatorname{dim} \widetilde{M}=2$ and $\varphi_{2}(\widetilde{M}) \subset S^{2}\left(c_{2}\right), c_{2}>0$.

3. $\varphi_{1}\left(M_{1}\right) \subset R^{3}$ and $\varphi_{2}$ has codimension 2. Therefore $\varphi_{1}\left(M_{1}\right) \subset S^{2}\left(c_{1}\right), \varphi_{2}$ is ppmc and $\varphi_{2}\left(M_{2}\right) \subset S^{2 m-1}\left(c_{2}\right)$ with $\frac{1}{c}=\frac{1}{c_{1}}+\frac{1}{c_{2}}$. Thus from theorem 1 , either $\operatorname{dim} M_{2}=2$ and $\varphi_{2}: M_{2} \rightarrow S^{3}\left(c_{2}\right)$ has constant mean curvature, or $\varphi_{2}\left(M_{2}\right)$ is an open subset of $S^{2}(a) \times$ $S^{2}(b) \subset S^{5}\left(c_{2}\right)$, where $\frac{1}{a}+\frac{1}{b}=\frac{1}{c_{2}}$.

$\mathbf{c}<\mathbf{0}$ As a model for the hyperbolic space we use the hyperbolic space model.

Now a new situation appears, namely when $C$ is totally umbilic and $m>2$. In this case we claim that $H$ is an umbilical direction of $\alpha$. To see this we consider a local orthonormal frame field $v_{1}, J v_{1}, \cdots, v_{m}, J v_{m}$ of $T M$ and, for each $1 \leq k \leq m$, we consider $V_{k}=\frac{1}{\sqrt{2}}\left(v_{k}-i J v_{k}\right)$. Clearly $C_{N}\left(V_{k}\right)=0$ and $C_{H}\left(V_{k}\right)=\lambda V_{k}$ for a certain real $\lambda$.

It follows from Gauss equation that

$$
C_{\alpha\left(X_{1}, X_{k}\right)} X_{j}=C_{\alpha\left(X_{j}, X_{k}\right)} X_{i} \quad, \quad \forall k, \forall i \neq j
$$


Therefrom $C_{\alpha\left(X_{1}, X_{k}\right)} X_{j}=0$ and $C_{\alpha\left(X_{j}, X_{k}\right)} X_{i}=0$. We also know that $\alpha\left(X_{i}, X_{k}\right)=\alpha^{H}\left(X_{i}, X_{k}\right) H+$ $\alpha^{N}\left(X_{i}, X_{k}\right) N$, where for $\xi \in C\left(T^{\perp} M\right), \alpha^{\xi}=\langle\alpha(.,),. \xi\rangle$.

The claim follows now from the fact that, $\forall i, k, \alpha^{H}\left(X_{i}, X_{k}\right)=0$, since $C_{\alpha\left(X_{1}, X_{k}\right)} X_{j}=$ $\alpha^{H}\left(X_{i}, X_{k}\right) C_{H} X_{j}=0$.

We may now conclude that $\varphi(M)$ is contained in a totally umbilical submanifold $W$ of $H^{2 m+2}(c)$ and that $\varphi: M \rightarrow W$ is minimal. Therefore we obtain one of the following situations:

$W=H^{2 m+1}(d)$; then $m=1$ and $\varphi: M \rightarrow H^{2 m+1}(d)$ is minimal.

$W=S^{2 m+1}(d)$; then $m=1$ and $M$ is minimal in $S^{2 m+1}(d)$.

$W=E^{2 m+1}$, a horosphere, so that $\varphi: M \rightarrow E^{2 m+1}$ is pluriminimal.

Assume now that $M=M_{1} \times M_{2}$, where trace $\alpha_{\mid T^{1} \times T^{1}} \neq 0$.

Using an adapted version of Lemma 1 [11] we know that $\varphi$ is a product immersion $\varphi_{1} \times \varphi_{2}$, that is, there exist two non-degenerate orthogonal affine subspaces $E_{1}$ and $E_{2}$ of the Minkoswski space $R_{1}^{2 m+3}$ such that $\varphi_{1}\left(M_{1}\right) \subset E_{1}$ and $\varphi_{2}\left(M_{2}\right) \subset E_{2}$. Now two cases must be considered: $\operatorname{dim} E_{1}=2 r_{1}+1$ or $\operatorname{dim} E_{2}=2 r_{1}+2$. We let $r_{2}=m-r_{1}$.

Assume first that $\operatorname{dim} E_{1}=2 r_{1}+2$. Then $\varphi_{1}\left(M_{1}\right)$ is contained either, in a hyperbolic space $H^{2 r_{1}}\left(c_{1}\right)$, or in an euclidean sphere $S^{2 r_{1}}\left(c_{1}\right)$, or in a horosphere $E^{2 r_{1}}$. Since $M_{1}$ is Kähler we end up with one of the following situations:

- $\varphi_{1}\left(M_{1}\right)$ is an open subset of $H^{2}\left(c_{1}\right)$ so that $\varphi\left(M_{2}\right) \subset S^{2 m-1}\left(c_{2}\right), \frac{1}{c_{1}}+\frac{1}{c_{2}}=\frac{1}{c}$, and $\varphi_{2}$ : $M_{2} \rightarrow S^{2 m-1}\left(c_{2}\right)$ is ppmc. Therefore it follows from theorem 1 that:

1. $\operatorname{dim} M_{2}=2$ and $\varphi_{2}: M_{2} \rightarrow S^{3}$ is ppmc.

2. $\varphi_{2}\left(M_{2}\right)$ is an open subset of $S^{2}(a) \times S^{2}(b) \subset S^{5}\left(c_{2}\right)$, where $\frac{1}{c_{1}}+\frac{1}{c_{2}}=\frac{1}{a}+\frac{1}{b}+\frac{1}{c_{2}}=\frac{1}{c}$, and we get $\varphi_{1}\left(M_{1}\right) \times \varphi_{2}\left(M_{2}\right) \subset H^{2}\left(c_{1}\right) \times S^{2}(a) \times S^{2}(b) \subset H^{8}(c)$.

- $\varphi_{1}\left(M_{1}\right)$ is an open subset of $S^{2}\left(c_{1}\right)$. We know then that $\varphi\left(M_{2}\right) \subset H^{2 m-1}\left(c_{2}\right), \frac{1}{c_{1}}+\frac{1}{c_{2}}=\frac{1}{c}$, and $\varphi_{2}: M_{2} \rightarrow H^{2 m-1}\left(c_{2}\right)$ is ppmc. From theorem 1 we infer that:

1. $\operatorname{dim} M_{2}=2$ and $\varphi=\varphi_{1} \times \varphi_{2}: M_{1} \times M_{2} \rightarrow S^{2}\left(c_{1}\right) \times H^{3}\left(c_{2}\right) \subset H^{6}(c)$.

2. $\varphi_{2}\left(M_{2}\right)$ is an open subset of $H^{2}(a) \times S^{2}(b) \subset H^{5}\left(c_{2}\right), \frac{1}{c_{1}}+\frac{1}{c_{2}}=\frac{1}{a}+\frac{1}{b}+\frac{1}{c_{2}}=\frac{1}{c}$, and $\varphi_{1}\left(M_{1}\right) \times \varphi_{2}\left(M_{2}\right) \subset S^{2}\left(c_{1}\right) \times H^{2}(a) \times S^{2}(b) \subset H^{8}(c)$.

3. $\varphi_{2}\left(M_{2}\right)$ is an open subset of a horosphere $E^{2 m-2}$ of $H^{2 m-1}\left(c_{2}\right)$. Then $\varphi_{1}\left(M_{1}\right) \times \varphi_{2}\left(M_{2}\right) \subset$ $S^{2}\left(c_{1}\right) \times E^{2 m-2} \subset S^{2}\left(c_{1}\right) \times H^{2 m-1}\left(c_{2}\right) \subset H^{2 m+2}(c)$.

- $\varphi_{1}\left(M_{1}\right)$ is an open subset of a horosphere $E^{2 m+1}$ of $H^{2 r_{1}+1}(c)$. Therefore $\varphi\left(M_{1} \times M_{2}\right) \subset$ $E^{2 m+1}$ and, from corollary 3 , we conclude that $\operatorname{dim} M_{1}=2, \varphi_{1}$ is $p p m c$ with image contained in a three-dimensional space and $\varphi_{2}$ is totally geodesic. 
- $\varphi_{1}\left(M_{1}\right)$ is an open subset of a horosphere $E^{2 r_{1}}$ of $H^{2 r_{1}+1}\left(c_{1}\right)$. In this case $\varphi_{2}\left(M_{2}\right) \subset$ $S^{2\left(m-r_{1}\right)}\left(c_{2}\right)$ and, since $M_{2}$ is Kähler, $\varphi_{2}\left(M_{2}\right)$ is an open subset of $S^{2}\left(c_{2}\right)$. Then $\varphi_{1}\left(M_{1}\right) \times$ $\varphi_{2}\left(M_{2}\right) \subset E^{2 m-2} \times S^{2}\left(c_{2}\right) \subset H^{2 m-1}\left(c_{1}\right) \times S^{2}\left(c_{2}\right)$.

Now if $\operatorname{dimE}_{1}=2 r_{1}+2, \varphi_{1}\left(M_{1}\right)$ is contained either, in a hyperbolic space $H^{2 r_{1}+1}\left(c_{1}\right)$, or in an euclidean sphere $S^{2 r_{1}+1}\left(c_{1}\right)$, or in a horosphere. So one of the following situations come out:

- $\varphi_{1}: M_{1} \rightarrow H^{2 r_{1}+1}\left(c_{1}\right)$ is ppmc and $\varphi_{2}\left(M_{2}\right) \subset S^{2\left(m-r_{1}\right)}\left(c_{2}\right)$, so that $r_{1}=m-1$ and $\varphi_{2}\left(M_{2}\right)$ is an open subset of $S^{2}\left(c_{2}\right)$. Then, from theorem 1, we get one of the following situations:

1. $\operatorname{dim} M_{1}=2$ and $\varphi=\varphi_{1} \times \varphi_{2}: M_{1} \times M_{2} \rightarrow H^{3}\left(c_{1}\right) \times S^{2}\left(c_{2}\right) \subset H^{5}(c), \frac{1}{c_{1}}+\frac{1}{c_{2}}=\frac{1}{c}$.

2. $\varphi_{1}\left(M_{1}\right)$ is an open subset of $H^{2}(a) \times S^{2}(b) \subset H^{5}\left(c_{1}\right), \frac{1}{a}+\frac{1}{b}+\frac{1}{c_{1}}=\frac{1}{c_{1}}+\frac{1}{c_{2}}=\frac{1}{c}$, and $\varphi_{1}\left(M_{1}\right) \times \varphi_{2}\left(M_{2}\right) \subset H^{2}(a) \times S^{2}(b) \times S^{2}\left(c_{2}\right) \subset H^{8}(c)$.

3. $\varphi_{1}\left(M_{1}\right)$ is an open subset of a horosphere $E^{2 m-2}$ of $H^{2 m-1}\left(c_{1}\right)$ and $\varphi_{1}\left(M_{1}\right) \times \varphi_{2}\left(M_{2}\right) \subset$ $E^{2 m-2} \times S^{2}\left(c_{2}\right) \subset H^{2 m+2}(c)$.

- $\varphi_{1}: M_{1} \rightarrow S^{2 r_{1}+1}\left(c_{1}\right)$ is ppmc and $\varphi_{2}\left(M_{2}\right)$ is an open subset of $H^{2}\left(c_{2}\right), \frac{1}{c_{1}}+\frac{1}{c_{2}}=\frac{1}{c}$. In this case we know from theorem 1 that one of the following situations hold:

1. $\operatorname{dim} M_{1}=2$ so that $\varphi=\varphi_{1} \times \varphi_{2}: M_{1} \times M_{2} \rightarrow S^{3}\left(c_{1}\right) \times H^{2}\left(c_{2}\right) \subset H^{6}(c)$.

2. $\operatorname{dim} M_{1}=4$ and $\varphi_{2}\left(M_{2}\right)$ is an open subset of $S^{2}(a) \times S^{2}(b) \subset S^{5}\left(c_{1}\right)$, so that $\varphi_{1}\left(M_{1}\right) \times$ $\varphi_{2}\left(M_{2}\right) \subset S^{2}(a) \times S^{2}(b) \times H^{2}\left(c_{2}\right) \subset H^{8}(c)$.

- $\varphi_{1}\left(M_{1}\right)$ is an open subset of a horosphere $E^{2 r_{1}+1} \subset H^{2 r_{1}+2}(c)$. In this case $\varphi\left(M_{1} \times M_{2}\right) \subset$ $E^{2 m+1}(c)$ and, from corollary 3 , we get $\operatorname{dim} M_{1}=2$ and $\varphi=\varphi_{1} \times \varphi_{2}$, where $\varphi_{1}$ is ppmc, $\varphi_{1}\left(M_{1}\right)$ is contained in a three-dimensional subspace of $E^{2 m+1}(c)$ and $\varphi_{2}$ is totally geodesic.

\section{Acknowledgments}

Work supported by ICTP, Trieste-Italy, CNPq, Brasil (Tribuzy) and FCT, Praxis XXI , Feder, project Praxis/2/2.1/MAT/125/94 (Ferreira). The authors would like to thank the Abdus Salam International Centre for Theoretical Physics, the International Atomic Energy Agency and Unesco for hospitality at the ICTP. They would like also to thank the Universidade do Amazonas and the University of Lisbon for all kind hospitality.

\section{References}

[1] Abe, K., On a Class of Hypersurfaces of $R^{2 n+1}$, Duke Math. J., 41 (1974), 865-874

[2] Burstall, F., Eschenburg, J., Ferreira, M. and Tribuzy, R. Kähler Submanifolds with Parallel Pluri-Mean Curvature, to appear 
[3] Dacjzer, M. and Gromoll, D., Real Kähler Submanifolds and Uniqueness of the Gauss Map, J. Diff. Geom.. 22 (1985), 13-25

[4] Dajczer, M. and Rodriguez, L., Rigidity of Real Kähler Submanifolds, Duke Math. J., 53 (1986), 211-220

[5] Eells and Lemaire, L., Another Report on Harmonic Maps, Bull. of London Math. Soc., 86, vol. 20 (1988)

[6] Erbacher, J. A., Reduction of the Codimension of an Isometric Immersion, J. Diff. Geom. 5 (1971), 333-340

[7] Eschenburg, J. H. and Tribuzy, R., Associated Families of Pluriharmonic Maps and Isotropy, Manuscripta Math. 95 (1998), 295-310

[8] Ferreira, M. and Tribuzy, R., Kählerian Submanifolds of $R^{n}$ with Pluriharmonic Gauss Map , Bull. Soc. Math. Belg. 45 ,Ser. B No2 (1993), 1183-197

[9] Ferreira, M. and Tribuzy, R., On the Type Decomposition of the Second Fundamental Form of a Kähler Manifold, Rend. Sem. Mat. Univ. Padova 94 (1995), 17-23

[10] Ferus, Symmetric Submanifolds of Euclidean Spaces, Math. Ann. 247 (1980), 81-93

[11] Moore, J. D., Isometric Immersions of Riemannian Products, J. Diff. Geom. 5 (1971), $159-168$

[12] Ryan, P., J., Kähler Manifolds as Real Hypersurfaces, Duke Math. J. 40 (1973), 207-213 